\title{
Aircraft noise generation and assessment: executive summary
}

\author{
L. Bertsch ${ }^{1}$ (1) $\cdot$ M. Snellen ${ }^{2} \cdot$ L. Enghardt ${ }^{3} \cdot$ C. Hillenherms ${ }^{4}$
}

Received: 8 February 2019 / Accepted: 12 February 2019 / Published online: 16 March 2019

(c) Deutsches Zentrum für Luft- und Raumfahrt e.V. 2019

\begin{abstract}
Based on the outcome of a workshop on aviation noise, this special issue on aircraft noise generation and assessment has been assembled. Invited contributions on preselected topics have been combined into one overall story line about both the aircraft noise generation and its assessment to provide an overview on the state-of-the-art capabilities in this research field (accounting both for modelling and measuring efforts). Although most relevant topics are covered, the editors do not claim that the collection of contributions is exhaustive for the multidisciplinary and vast research area dealing with aircraft noise. This issue is not restricted to current aircraft technology only, but includes low-noise technologies and novel aircraft design for subsonic and supersonic vehicles. Furthermore, an overview on measurement techniques, aeroacoustic windtunnel design, and low-noise modifications to existing windtunnels is provided. The selected paper contributions are all peer reviewed and display the status quo of experimental and numerical research activities in academia, at research institutes, and in industry.
\end{abstract}

Keywords Executive summary $\cdot$ CEAS special edition $\cdot$ Aircraft noise generation $\cdot$ Aircraft noise assessment

\section{Preamble}

In October 2014, a workshop in the field of "Aviation Noise" was organized in a joint effort by DLR \& TU Delft ${ }^{1}$. The specific topic of this workshop was "Aircraft Noise Reduction at the Source" for conventional tube-and-wing aircraft. Specific tasks were predefined to be addressed in the workshop:

- identification of the main noise sources on-board of a reference vehicle;

- assessment of current simulation capabilities (status 2014);

This editorial is part of a Special Issue on Aircraft Noise Generation and Assessment.

\section{Bertsch}

lothar.bertsch@dlr.de

1 DLR, Institute of Aerodynamics and Flow Technology, Göttingen, Germany

2 Delft University of Technology, Section Aircraft Noise and Climate Effects, Delft, The Netherlands

3 DLR, Institute of Propulsion Technology, Berlin, Germany

4 CEAS Aeronautical Journal, and DLR, Institute Development Aeronautics, Cologne, Germany
- identification and assessment of promising noise reduction concepts;

- assessment of the overall effect of these concepts if installed and operated on-board of an aircraft.

Finding an answer to these questions requires a multidisciplinary and broad approach and the involvement of participants with different backgrounds, bringing their individual ideas and concepts to the table. Therefore, known experts from industry, academia, and universities were invited to share their knowledge. Instead of having yet another presentation marathon, the participants were assigned into small working groups to enable a focused and in-depth discussion. To avoid a biased outcome from established groups, diversity in background was aimed for and as such direct colleagues were assigned to different working groups as much as possible. Ultimately, the results of each individual working group were presented and discussed in plenary sessions to find common ground and identify the most promising concepts and ideas.

The outcome of the 2014 workshop [1] is an overview of noise generating mechanisms, relevant input parameters, and interaction effects for the most relevant noise sources on-board of conventional mid-range transport aircraft equipped with conventional turbofan engines, see Fig. 1. The

${ }^{1}$ First Joint DLR \& TU Delft Aviation Noise Workshop, 6-8 Oct 2014, Meisdorf, Harz, Germany. 
Fig. 1 Depiction of most relevant noise sources on-board of conventional mid-range transport aircraft, (C) Henri Siller and Jan Delfs, DLR, 2019

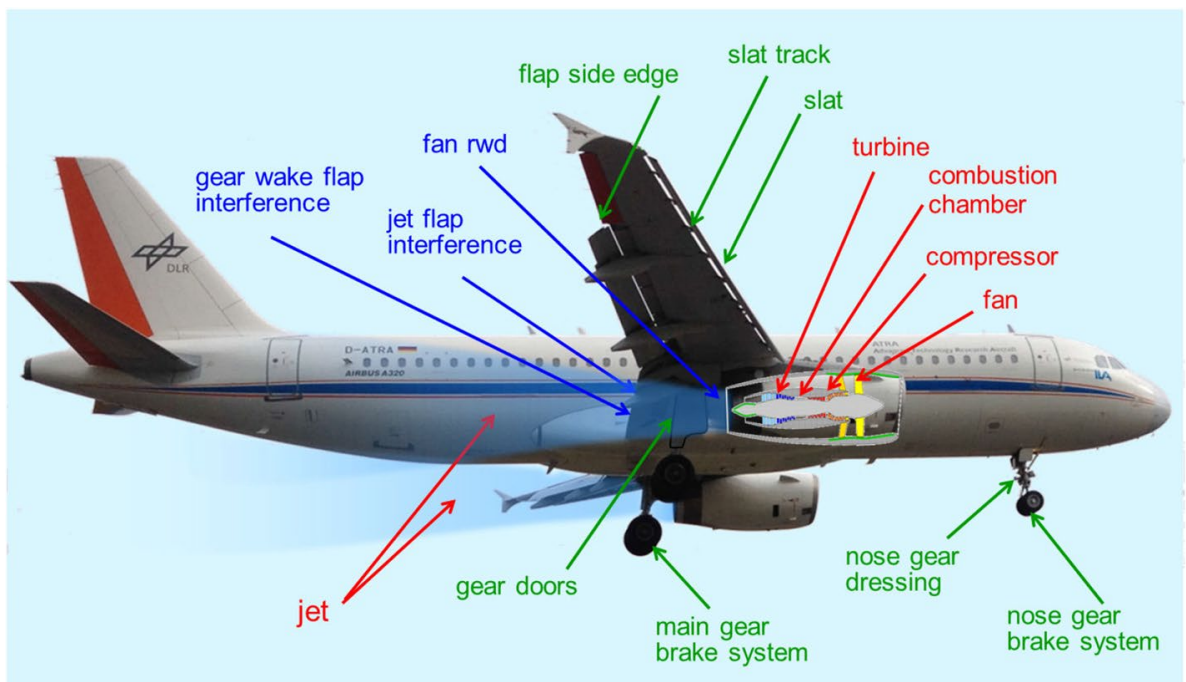

participants, furthermore, identified promising noise reduction concepts for each of these noise sources. The impact of each of the reduction concepts on the overall system noise was assessed and quantified. These level reductions are based on the individual assessment of the participants and reflect their experience and engineering judgement. Finally, a classification of available simulation tools in the field of aviation noise was derived. The most commonly accepted tools were listed and categorized accordingly [1].

To confirm the findings of the 2014 workshop on a scientific level, detailed experimental, numerical, and theoretical studies are essential; hence, the idea of a joint and peer reviewed publication was born. In the following sections, a brief description of each selected contribution is presented and the overall special edition is briefly summarized.

\section{Contents of the special edition}

The special edition is comprised of five chapters, i.e., (1) airframe noise, (2) engine noise, (3) installation effects, (4) assessment techniques, and (5) overall aircraft simulation. The chapter selection is based on the 2014 workshop findings [1] with the overall goal to provide a broad overview on the generation and the assessment of aircraft noise. A list of topics and associated experts has been assembled from the workshop participants, and missing areas have been identified and included, i.e., windtunnel design, measurement techniques, supersonic flight, and a general description of aircraft noise assessment and the relevant noise descriptors. Authors have been invited to contribute with a preselected and specific topic according to their expertise. To ensure the inclusion of state-of-the-art insights, the main authors have invited other experts from the research field as co-authors. Each contribution to the special edition also presents a current overview of the most relevant activities in the specific research field. Compared to other special editions, invited authors contribute to predefined topics to realize an overall storyline and to avoid a random collection of separate papers.

The first two chapters focus on typical airframe and engine noise sources of today's turbofan driven aircraft. The main focus lies on simulation and measurement of selected noise sources. In addition, promising technologies and design concepts to further reduce the engine and airframe noise contribution are discussed. Chapter 3 is dedicated to the noise-related effects caused by the interaction of certain aircraft components. These so-called installation or interaction effects can be either advantageous or disadvantageous with respect to the overall noise. The chapter includes recent developments in simulating, measuring, and exploiting these installation effects towards novel aircraft configurations. Chapter 4 is dedicated to experimental techniques to measure both component noise emission and overall aircraft noise. In addition, contributions on dedicated low-noise facilities and on modifications to adapt an existing windtunnel for aeroacoustic measurements are included in this chapter. Chapter 5 focuses on the overall aircraft noise as received on the ground. Furthermore, a contribution is dedicated to supersonic aircraft, because supersonic transportation currently seems to experience a renaissance with multiple companies working on high-speed business jets.

\subsection{Chapter 1: Airframe Noise}

Airframe components have more and more become a major contributor to the overall aircraft noise. Ever since engine bypass ratios have initially been increased in the 1970s, the role of airframe noise became more prominent. During typical approach flights, airframe noise can nowadays 
even dominate the overall aircraft noise as received on the ground. To reduce airframe noise, a thorough understanding of the inherent mechanisms behind the noise generation becomes essential. Numerical and experimental ground work is required to capture and understand the physical mechanisms. Only if the physics is fully understood, one can develop adequate modeling and thus prediction capabilities. The availability of such capabilities is essential to establish appropriate reduction technologies. The chapter on airframe noise is comprised of four contributions, i.e., Refs. [2-5], that are briefly summarized here.

\subsubsection{Airframe noise modeling and prediction-by N. Molin}

Ref. [2] presents a review of methods for airframe noise analytical modeling and prediction, as used by aircraft manufacturers for a rapid assessment of noise exposure around airports, certification noise levels, and optimized operational procedures at various stages of aircraft design. At present, these kinds of noise models are based on analytical or semiempirical modeling. The paper presents the main noise mechanisms for slats, flaps and landing gears, as well as the associated noise models used for an airframe noise prediction. These models were validated with measurements performed during specific noise flight tests on various Airbus aircrafts, as shown in the paper.

\subsubsection{Towards noise certification during design: airframe noise simulations for full-scale, complete aircraft-by M. Khorrami and E. Fares}

The advancement of simulation-based, system-level, airframe noise prediction tools is important for the development of quiet commercial aircraft. A recent, NASAsponsored effort is summarized in Ref. [3]. A systematic approach was followed to extend current computational tools to a full-scale, landing aircraft under realistic flight conditions. The work involved several phases: large-scale, highfidelity, unsteady flow simulations; model-scale experiments in ground-based facilities; and farfield noise prediction for a full-scale, complete aircraft. Excellent agreement between model-scale measured and simulated data promoted application of the computational methodology to full-scale configurations. Good agreement with flight test data was obtained for the simulated farfield noise.

\subsubsection{Limits and challenges of aircraft retro-fitting for noise reduction-by M. Pott-Pollenske}

As many aircrafts are operated over decades, a short-term noise reduction is not a matter of new aircraft developments but strongly targeting the question how to reduce the noise generated by the existing aircraft fleet. Since the acoustic benefit of operational means is limited, a source noise reduction is the most promising way to achieve meaningful noise reductions. As is obvious, all kinds of retro-fit means to reduce airframe and engine-related noise needs to undergo the typical and precisely defined procedure of certification or even qualification, in case only a flight test demonstration is envisaged. In Ref. [4], selected limits and challenges will be described that occur during this procedure and that need to be incorporated in the noise reduction device's design and application.

\subsubsection{A flight demonstration for airframe noise reduction technology-by K. Yamamoto, K. Hayama, T. Kumada, and K. Hayashi}

Ref. [5] overviews activities and results for an initial flight demonstration of a project, "FQUROH", to develop airframe noise reduction technologies that utilize advanced Computational Fluid Dynamics (CFD). For the initial demonstration held in 2016, noise reduction concepts for the flap and main landing gear were successfully applied to the actual complex geometries of a research aircraft using unsteady CFD and aeroacoustic wind tunnel testing. The flight test of the modified aircraft was carried out with 177 noise source measurements, and provided encouraging results showing feasibility of the noise reduction technologies.

\subsection{Chapter 2: Engine Noise}

Combustion engines have always been among the most dominating noise sources on-board of any motorized and flying vehicle, i.e., independent of the flight situation. For conventional turbofan engines, the overall engine noise is dominated by fan and jet noise. In the last decades, the dominance of these two sources has been prominent, but also begun to decrease slightly, i.e., especially due to increased engine bypass ratios and advanced fan design. Although noise reduction has not been the primary concern behind these modifications, improving engine performance has helped to reduce the noise generation of fan and jet as well. Increased bypass ratios of the engine have reduced jet exhaust velocities, hence significantly decreased jet noise generation. Advanced design of fan blades and rotor-stator arrangement has reduced the corresponding noise generation. Implementation of specifically designed acoustical lining concepts further reduced fan noise emission. Yet, other inherent noise sources of the engine are still masked by the dominating jet and fan contribution, i.e., noise generated by the engine core is still less relevant in the context of overall engine noise. Still, if jet and fan noise are further reduced due to advanced design or during a specific operating condition, e.g., flight idle, the engine core can have an impact 
on the overall engine noise. The chapter on engine noise is comprised of three contributions, i.e., Refs. [6-8], that are briefly summarized here.

\subsubsection{Current engine noise and reduction technology—by E. Nesbitt}

Ref. [6] reviews current state of the art of turbofan engine noise and engine noise reduction technology, specifically focusing on the engine technology of larger passenger jetliners which have entered into service in the 2007 through 2017 timeframe. Important factors in turbofan engine design from a community noise perspective, and the sources of noise along with their relative importance are also presented. A review of different engine noise reduction technologies is part of this contribution, as well as an estimate of the technology readiness level. Finally, potential tradeoffs, challenges, and future technology directions are outlined.

\subsubsection{Combustion noise: modeling and prediction - by C.K.W. Tam, F. Bake, L.S. Hultgren, and T. Poinsot}

Ref. [7] reviews both direct and indirect combustion noise. Because of the complexities inherent in combustion processes, a widely accepted combustion noise theory has yet to be developed. For this reason, this review focuses primarily on experimental measurements, semi-empirical relations and empirical but practical prediction methodologies. Generation of indirect combustion noise by the passage of entropy waves through a non-uniform mean flow was predicted in the 1970s. Recently, its existence was confirmed experimentally. This was followed by numerous publications on this subject. They form the primary materials of this review. Recent advances in computational methods lead us to envision an important role to be played by high-fidelity numerical simulation in future combustion and entropy noise research and prediction.

\subsubsection{Advanced liner concept-by F. Bake and K. Knobloch}

To address the demand of modern low-emission aeroengines for improved acoustic damping in a broadband frequency range, two different novel acoustic liner concepts are introduced in Ref. [8]. The first one, called Zero-Massflow Liner (ZML), enhances the acoustic damping of a liner by inducing an additional periodic in- and outflow through the liner perforation. It is generated by an independent acoustic excitation of the liner cavity. The additional excitation is not tied to the frequency of the target sound field which shall be damped, which is an outstanding feature compared to other active noise reduction methods. The other liner concept (FlexiS) increases the broadband damping characteristics of the classical Single-Degree-of-Freedom liner by introducing flexible wall structures which on one hand deliver further degrees of freedom and on the other hand also provide additional intrinsic damping properties of the flexible wall material. A proof of concept study for both liner concepts is presented.

\subsection{Chapter 3: Installation Effects}

Installation effects directly influence the noise generation, result in additional and new noise sources, or change the emission directivity of a noise source. These effects can significantly decrease the overall aircraft noise; hence, profound knowledge and understanding of these effects is of utmost importance. The 2014 workshop participants have identified structural noise shielding as one of the most promising technologies to further reduce fan noise [1]. All known and published approaches towards low-noise aircraft design incorporate fan noise shielding. Obviously, a thorough assessment to understand the involved physics is a prerequisite for a proper and effective design. The chapter will present recent developments in simulating, measuring, and exploiting these installation effects towards novel aircraft configurations. The chapter on installation effects is comprised of 3 contributions, i.e., Refs. [9-11], that are summarized here.

\subsubsection{Advanced Low Noise Aircraft Configurations and Their Assessment-Past, Present and Future-by Z.S. Spakovszky}

Novel aircraft concepts and architectures, enabled by distributed, more integrated, and boundary layer ingesting propulsion systems, pose new aeroacoustic problems which require innovative approaches. The Silent Aircraft Initiative (SAI) was aimed at the conceptual design of an aircraft imperceptible to the human ear outside the airport perimeter. A decade past SAI novel aircraft architectures, such as the D8 double bubble aircraft, electric-, hybrid-, and turbo-electric aircraft concepts are being investigated as potential game changers. Ref. [9] discusses a selection of enabling technologies and gives perspectives on future trends and new directions in aeroacoustics required to address the challenges.

\subsubsection{Noise shielding—numerical investigation-by $M$. Lummer}

Acoustic installation effects are considered as a scattering problem. Two methods to investigate them are presented in Ref. [10]. First, a Fast Multipole Method-Boundary Element Method (FMM-BEM) which can solve the Helmholtz wave equation for full-scale aircraft configurations at frequencies of some kHz. There, low Mach number potential mean flow fields can be taken into account by a so-called Taylor transformation. Second, a Discontinuous Galerkin Method 
(DGM) which solves Acoustic Perturbation Equations (APE) for realistic mean flow fields is introduced. DGM calculations are very expensive and can be performed for full-scale aircrafts only at low frequencies. Its main purpose so far is to assess the accuracy of the FMM-BEM for selected cases. Besides generic examples, scattering results for a 1 $\mathrm{kHz}$ monopole at a full-scale aircraft geometry are given.

\subsubsection{Validation Data for Aircraft Noise Shielding Prediction-by K.-S. Rossignol, J. W. Delfs, M. Moessner, M. Lummer, and J. Yin}

The design of future low-noise aircraft will require noise shielding as a fundamental design feature. This aspect was already recognized in the seventies, where early experimental work set the ground on possible solutions to the conventional, short and reduced take-off and landing noise problem. Current low-noise aircraft design concepts of the hybrid-wing-body type require establishing a new knowledge base to allow an assessment of their potential noise benefit. Ref. [11] presents an overview of recently acquired datasets from dedicated experimental investigations on acoustic shielding, ranging from simple generic test cases to full 3D aircraft configurations, as well as comparisons with numerical results. Results from experiments on a wide range of configurations are presented, providing an extensive high-quality validation database for numerical prediction methods.

\subsection{Chapter 4: Assessment Techniques}

The focus of this chapter lies on experimental techniques to measure both component noise emission and overall aircraft noise. Novel measurement techniques have to be applied to separate and identify underlying noise sources and mechanisms from a measured signal. This is important to develop an in-depth understanding of the physics behind the noise generation. Moreover, experimental data of high quality are the key requirement to validate any numerical prediction. In that context, if measurements are taken in a test facility, it is important to understand the requirements and implications of such an "in-door" experiment. The chapter on assessment techniques is comprised of four contributions, i.e., Refs. [12-15], that are summarized here.
2.4.1 A review of acoustic imaging methods using phased microphone arrays-by R. Merino-Martinez, P. Sijtsma, M. Snellen, T. Ahlefeldt, J. Antoni, C. J. Bahr, D. Blacodon, D. Ernst, A. Finez, S. Funke, T. F. Geyer, S. Haxter, G. Herold, X. Huang, W. M. Humphreys, Q. Leclere, and A. M. N. Malgoezar

Phased microphone arrays have become a well-established tool for performing aeroacoustic measurements in wind tunnels (both open-jet and closed section), flying aircraft and engine test beds. Ref. [12] provides a review of the most well known and state-of-the-art acoustic imaging methods with a consistent nomenclature and extensive references. Practical recommendations on when to use each method are also included. Moreover, several exemplary results showing and comparing the performance of most methods in aeroacoustic applications are presented. This contribution provides a general introduction to aeroacoustic measurements for non-experienced microphone array users as well as a broad overview for general aeroacoustic experts.

\subsubsection{Aeroacoustic windtunnel design—by J. Gomes, A. Bergmann, and $\mathrm{H}$. Holthusen}

Further progress in airframe noise research requires aeroacoustic wind tunnels with extremely low background noise and pressure fluctuations. Ref. [13] discusses the design of modern aeroacoustic wind tunnels. The approach discussed in this contribution subdivides the wind tunnel design into different sections according to the multitude of solutions and design tools that are required to complete it. Thus, while the design of the airline circuit and drive unit is strongly based on coupled numerical solutions of CFD and acoustic solvers, the design of the acoustic plenum gives more emphasis to in situ observations and to experimental results.

\subsubsection{Exploitation of hybrid anechoic wind tunnels for aeroacoustic and aerodynamic measurements- by K. A. Brown, W. J. Devenport, and A. Borgoltz}

The last 10 years have seen the establishment of the hybrid anechoic wind tunnel as a significant tool for experimental aeroacoustics research. The hybrid anechoic arrangement offers a number of advantages over the traditional open-jet test section, including low interference corrections that allow for testing of large models at higher Reynolds numbersadvantages well suited to aircraft noise source research. In Ref. [14], recent applications of hybrid anechoic tunnels for aircraft noise source studies are reviewed. The authors discuss the established practice for acoustic and aerodynamic corrections of measurements performed in these facilities, and assess some of the latest findings in aerodynamic behavior of hybrid anechoic tests sections. 


\subsubsection{Comparison of microphone array measurements in the closed test section of LSWT and ETW-by C. Spehr and T. Ahlefeldt}

In Ref. [15], a comparison of microphone array measurements using the very same aircraft half-model in a closed test section of two different wind tunnels with two different microphone arrays is made, giving an insight in the repeatability of industrial array measurements. The comparison is made by juxtaposing the two measurements in terms of source maps at individual frequencies and in terms of integrated spectra for different areas of the wing. It showed substantial agreement but yet noticeable differences which are discussed afterwards taking into account the model and model support, background noise, flow conditions, microphone array setup, and post processing.

\subsection{Chapter 5: Overall Aircraft Simulation}

Chapter 5 focuses on the overall aircraft noise as received on the ground. Here, the overall aircraft as a sum of all its subcomponents under realistic operating conditions is under investigation. Basic descriptors of overall aircraft noise are introduced and available simulation approaches towards overall aircraft noise are presented. The focus of all contributions lies on the physical effects and not on psychological implications of the overall aircraft noise, i.e., pychoacoustics and related disciplines are not explicitly considered here. Yet, the technique of auralization, i.e., part of Chapter 5, promises to close the gap between physics and psychology in the near future. Finally, a dedicated contribution on supersonic aircraft can be found in Chapter 5. The chapter on overall aircraft simulation is comprised of three contributions, i.e., Refs. [16-18], and is summarized here.

\subsubsection{Aircraft noise immission modeling-by U. Isermann and L. Bertsch}

Ref. [16] focuses on the simulation of the aircraft noise received on the ground. This process includes the description of the sound emission by the aircraft and the modeling of the sound propagation through the atmosphere. An overview is provided on how aircraft noise immission can be described and assessed by noise descriptors. Fundamentals of aircraft noise modeling are explained, including a classification of aircraft noise models into best practice and scientific models and their applicability to noise mitigation measures. Furthermore, the overall workflow of the task of noise modeling is explained as well the special role of noise model databases and the simulation of aircraft flightpaths. The most common methods to describe the sound propagation through the atmosphere are introduced.

\subsubsection{Auralization of air vehicle noise for community noise assessment-by S. Rizzi and A. Sahai}

Ref. [17] serves as an introduction to air vehicle noise auralization and documents the current state-of-the-art. Auralization of flyover noise considers the source, path, and receiver as part of a time-marching simulation. Two approaches are offered; a time-domain approach performs sound synthesis followed by propagation, while a frequency-domain approach performs propagation followed by sound synthesis. Source noise description methods are offered for isolated and installed propulsion system and airframe noise sources for a wide range of air vehicles. Methods for synthesis of broadband noise, discrete tones, steady and unsteady periodic, and aperiodic sources are presented, and propagation methods and receiver considerations are discussed. Auralizations applied to vehicles ranging from large transport aircraft to small unmanned aerial systems demonstrate current capabilities.

\subsubsection{Overall Vehicle System Noise: Sonic Boom—by J. Page and $A$. Loubeau}

Worldwide interest in civilian supersonic flight has increased substantially over the last decade. Ref. [18] describes recent international research on low-boom aircraft design, propagation modeling and human subjective response. The authors are actively conducting research in this area, and with contributions from the Japanese Aerospace Exploration Agency (JAXA), Lockheed Martin, Aerion Corp., Gulfstream Aerospace Corp. and Eagle Aeronautics have provided information about current research and preparation of information for potential international supersonic overland flight rule changes.

\section{Summary}

Based on the outcome of a joint DLR and TU Delft Aviation Noise Workshop, this special edition on aircraft noise has been assembled: "Aircraft Noise Generation and Assessment". The variety of topics addressed in this special issue indicates the multidisciplinarity of the research field and as such highlights the need for a collective effort from within the research community and industry to work towards more silent aircraft. Both in the field of modelling and measurements, different levels of complexity can be discerned. Models range from highly detailed, but computationally expensive numerical simulations to semi-empirical models and in a next level to the models based on tables of noise levels. Measurements range from windtunnel measurements investigating the aircraft noise on a component level to measurements taken at airports of aircraft under 
operational conditions. It is important to ensure that the insights from the more detailed modelling and measurement efforts find their way towards the more computational efficient approaches. Furthermore, insights obtained during windtunnel measurements can be used to optimize the measurements and analysis of full aircraft. Similarly, the interaction between the fields of modelling and measuring is essential. Models need validation and the analysis of the measurements can benefit from insights obtained through modelling. The guest editors of this special issue aim to contribute to the above-mentioned interactions and to provide a comprehensive overview of the state-of-the-art capabilities in this research field by the collection of high-quality contributions of internationally recognized experts.

Acknowledgements The Editors thank all authors of the contributions and express their appreciation to the participants of the First joint DLR \& TU Delft Aviation Noise Workshop for their valuable input [1] which formed the basis and created the network of experts to initiate this special issue.

\section{References}

1. Bertsch, L., Simons, D., Snellen, M.: Aircraft noise: the major sources, modelling capabilities, and reduction possibilities. German Aerospace Center \& Delft Technical University, DLR IB 224-2015 A 110

\section{Chapter 1: Airframe Noise}

2. Molin, N.: Airframe noise modeling and prediction. CEAS Aeronaut. J. (2019). https://doi.org/10.1007/s13272-019-00375-4

3. Khorrami, M., Fares, E.: Toward noise certification during design: airframe noise simulations for full-scale, complete aircraft. CEAS Aeronaut. J. (2019). https://doi.org/10.1007/s13272-019-00378-1

4. Pott-Pollenske, M.: Limits and challenges of aircraft retrofitting for noise reduction. CEAS Aeronaut. J. (2019). https://doi. org/10.1007/s13272-019-00370-9

5. Yamamoto, K., Hayama, K., Kumada, T., Hayashi, K.: A flight demonstration for airframe noise reduction technology. CEAS Aeronaut. J. (2019). https://doi.org/10.1007/s13272-019-00376-3

\section{Chapter 2: Engine Noise}

6. Nesbitt, E.: Current engine noise and reduction technology. CEAS Aeronaut. J. (2019). https://doi.org/10.1007/s13272-019-00381-6
7. Tam, C.K.W., Bake, F., Hultgren, L.S., Poinsot, T.: Combustion noise: modeling and prediction. CEAS Aeronaut. J. (2019). https ://doi.org/10.1007/s13272-019-00377-2

8. Bake, F., Knobloch, K.: Novel liner concept. CEAS Aeronaut. J. (2019). https://doi.org/10.1007/s13272-019-00380-7

\section{Chapter 3: Installation Effects}

9. Spakovszky, Z.S.: Advanced low noise aircraft configurations and their assessment: past, present and future. CEAS Aeronaut. J. (2019). https://doi.org/10.1007/s13272-019-00371-8

10. Lummer, M.: Installation - numerical investigation. CEAS Aeronaut. J. (2019). https://doi.org/10.1007/s13272-019-00382-5

11. Rossignol, K.-S., Delfs, J.W., Moessner, M., Lummer, M., Yin, J.: Validation data for aircraft noise shielding prediction. CEAS Aeronaut. J. (2019). https://doi.org/10.1007/s13272-019-00387-0

\section{Chapter 4: Assessment Techniques}

12. Merino-Martinez, R., Sijtsma, P., Snellen, M., Ahlefeldt, T., Antoni, J., Bahr, C.J., Blacodon, D., Ernst, D., Finez, A., Funke, S., Geyer, T.F., Haxter, S., Herold, G., Huang, X., Humphreys, W.M., Leclere, Q., Malgoezar, A.M.N.: A review of acoustic imaging methods using phased microphone arrays. CEAS Aeronaut. J. (2019). https://doi.org/10.1007/s13272-019-00383-4

13. Gomes, J., Bergmann, A., Holthusen, H.: Aeroacoustic wind tunnel design. CEAS Aeronaut. J. (2019). https://doi.org/10.1007/ s13272-019-00372-7

14. Brown, K.A., Devenport, W.J., Borgoltz, A.: Exploitation of hybrid anechoic wind tunnels for aeroacoustic and aerodynamic measurements. CEAS Aeronaut. J. (2019). https://doi. org/10.1007/s13272-019-00385-2

15. Spehr, C., Ahlefeldt, T.: Comparison of microphone array measurements in the closed test section of LSWT and ETW. CEAS Aeronaut. J. (2019). https://doi.org/10.1007/s13272-019-00386-1

\section{Chapter 5: Overall Aircraft Simulation}

16. Isermann, U., Bertsch, L.: Aircraft noise immission modeling. CEAS Aeronaut. J. (2019). https://doi.org/10.1007/s13272-01900374-5

17. Rizzi, S.A, Sahai, A.K: Auralization of air vehicle noise for community noise assessment. CEAS Aeronaut. J. (2019). https://doi. org/10.1007/s13272-019-00373-6

18. Page, J., Loubeau, A.: Overall vehicle system noise: sonic boom. CEAS Aeronaut. J. (2019). https://doi.org/10.1007/s13272-01900379-0 